\title{
Installation Of Black Box In Automobile
}

\author{
ShantanuNandurkar, Sameer Khirpurikar, Sanket Dixit, Sachin Rathi, Samrudha \\ Karangale \\ 54, prakash nagar, near ekvira vidyut colony, behind navodaya vidyalaya, Amravati \\ 305, mayureshwar aparatment, tower line, v.m.v. road, Amravati
}

\begin{abstract}
Most people associate black boxes with airplanes but they are no longer just the key tool in investigation of airplane accidents. The event data recorder (EDR) as the black box is officially called is slowly gaining an important role in investigation of car accidents as well. Despite the fact that some people have expressed concerns about privacy issues, car black boxes are not helpful for car accident investigators and insurance companies only. By recording the events and actions of the driver including speed, braking, turning, etc. seconds before the collision, the car black box will undoubtedly help both the police and insurance companies in reconstruction of the events before the accident but it will also bring a number of benefits for the car's owner. In our project we focused on some parameters of cars which may be helpful to find out the real cause of the accident or to have a brief idea about the accident. The main parts of the black box are Event Data Recorder (EDR), Crash Survival Memory Unit (CSMU).
\end{abstract}

Keywords: Crash Survival Memory Unit (CSMU)t, Event Data Recorder(EDR), Memory unit, Video Camera, Power Supply.

\section{Introduction}

As often the black box are related to airplanes only, but nowadays the period has come to have a special Black Box in automobile because the number of accident of automobiles are gradually increases over period of time. BLACK BOX is a device which is used to record the parameters of a vehicle. Information from these devices can be collected after a crash and analyzed to help determine what the vehicles were doing before, during and after the crash or event. Various types of sensors are employed to record the information.

\subsection{DEFINATION OF BLACK BOX}

Black Box is a device which is used to record the parameters of a vehicle. Information from these devices can be collected after a crash and analyzed to help determine what the vehicles were doing before, during and after the crash or event.

\subsection{NEED OF BLACK BOX}

As there are many advantages of installing black box in cars. Automotive black boxes are different than the kind that is in airplanes, which continuously record audio, mechanical functions and location. In cars, the box only transmits information to the car's computer in the event of a crash or air bag deployment. Recorders capture vehicle speed, brake activity, crash forces and even seatbelt use at the time of the event.

Many vehicles already have car black box but most people are not aware that their car contains the device that works similar to those found in airplanes. The first event data recorder in car was installed already in the mid1990; while up to $85 \%$ of new vehicles have one behind the dashboard or under the seat. They do not record voices and do not violate the car's owner privacy in any way despite the fact that some people claim that they feel like being spied on. These types of car black boxes do not track the car's movement or the driver's actions all the time but they do record several types of important data few seconds before the collision. This is due to the fact that these devices were originally designed to monitor activation of the airbags. For that reason the device is activated about few seconds before the actual collision.

The data recorded by a car black box is also very helpful for insurance claims and there is no way to be rejected with the evidence from the black box. Even more, you may even get a discount for your car insurance if using a car black box. The data recorded by black boxes that are already installed in your vehicle is very strong evidence as well but without a video record, you may not be able to prove that a cyclist suddenly turned in front of your car without any signal.

\subsection{WHAT IS AUTOMOBILE BLACK BOX?}

Black Box is a device installed in some automobiles to record information related to vehicle crashes or accidents. It is also called EDR (Event Data Recorder)[1]. In modern diesel trucks, EDRs are triggered by electronically sensed problems in the engine (often called faults), or a sudden change in wheel speed. One or 
more of these conditions may occur because of an accident. Information from these devices can be collected after a crash and analyzed to help determine what the vehicles were doing before, during and after the crash or event.

\subsection{PRINCIPLE OF AUTOMOBILE BLACK BOX}

The basic principle of the black box is to collect the data from the various sensors install in EDR and stored the record data in the memory unit. The storage unit of the black box works so that it always store the data of few seconds( $70-80 \mathrm{sec}$ ) before the crash. By retrieving these data we can plot the graph and by studying that graph and analyzing helps us to predict the cause of the car accident. Also it contains the video recorder (not audio) to record the view of road[3].

\section{Architecture Of Event Data Recorder}

An Event Data Recorder or EDR is a device install in some automobile to record information related to vehicle crashes or accidents. EDRs are triggered by electronically sensed problems in the engine (often called faults), or a sudden change in wheel speed. [5]One or more of these conditions may occur because of an accident. Information from these devices can be collected after a crash and analyzed to help determine what the vehicles were doing before, during and after the crash or event.

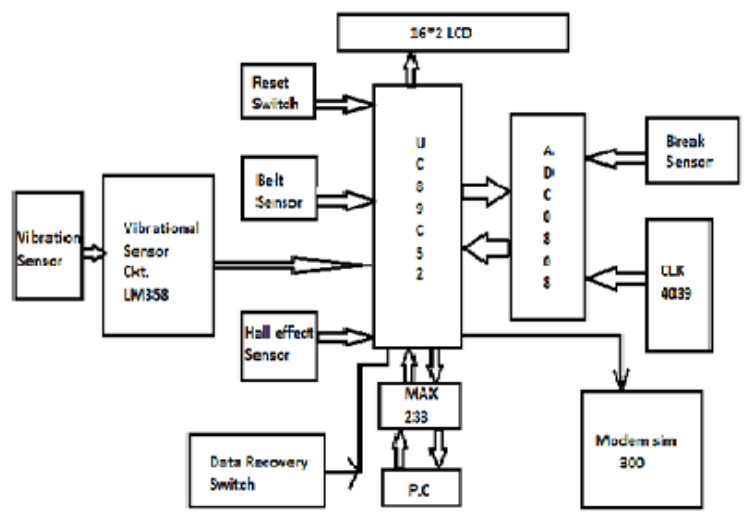

Fig.1 Architecture of EDR

1. Speed sensor: - it is mainly used to record the speed of the vehicle for this we have used the Hall Effect sensor, with the help of this sensor we can measure the RPM of wheel, from which we can easily determine the speed of vehicle before accident. The assembly of speed sensor consists of mainly Hall Effect sensor and magnet. The magnet is attached on the wheel and the Hall Effect sensor is fixed at a closed position of the wheel. Hall Effect senses the magnetic field produced by magnet. Due to rotation of wheel there is continuous change in magnetic field this change in magnetic field senses by the Hall Effect sensor and it creates output which generates the pulse, an d converted into the RPM of the wheel.

2. Seat belt switch: - This switch is used to determine whether the driver fasten the seat belt during the driving or not. This is simply on/off switch which generate only two output 0 and 1.0 when seat belt is not fastening and 1 when it is fasten. The seat belt sensor is simply consisting of On/Off switch, which has two positions on and off.

3. Brake Sensor: - This sensor gives the information about the amount of brake applied by driver. This sensor consist of the three level of sensation, it will sense the level at which the brake pedal is applied. Like it has level 1 , level 2, level 3, increase in level indicates the more pressure applied by driver on the brake pedal.

4. Vibration sensor: - Vibration sensor basically consists of Quad crystal. When voltage is providing to the Quad crystal as input it generate vibration as output and vice versa. When accident happened vibration generated inside the automobile act as a input to the vibration sensor which result in the generation of voltage as a output. 5. Memory Unit :-Flash Memory: Flash is the most important recent advancement in memory technology. It combines all the best features of the memory devices described thus far. Flash memory devices are high-density, low-cost, nonvolatile, fast (to write but not to Read), and electrically reprogrammable. These advantages are overwhelming, and the use of flash memory has increased dramatically in embedded systems as a direct result. 6. Video Camera:-In the case of video cameras a series of individual pictures are captured and then presented in rapid succession to give the illusion of having captured motion. In this way we can have the actual video recording of the accident from the drivers view.

7. Power Supply:- The target board is powered by a 5v power supply. The power supply is designed such that, an $\mathrm{AC}$ or DC voltage from range $7 \mathrm{~V}$ to $24 \mathrm{~V}$ may be given as input. The components used in supply design. Circuit supplies constant voltage regardless of changes in load current by Regulator. 


\section{Design of Crash Survival Memory Unit (CSMU)}

The CSMU in a solid-state black box insulates and protects the stack of memory boards that store the digitized information [8].

Aluminum housing - There is a thin layer of aluminum around the stack of memory cards.

High-temperature insulation- This dry-silica material is $1 \mathrm{~cm}$ thick and provides high-temperature thermal protection. This is what keeps the memory boards safe during post-accident fires.

Stainless-steel shell- The high-temperature insulation material is contained within a stainless-steel cast shell that is about 0.25 inches $(0.64 \mathrm{~cm})$ thick. Titanium can be used to create this outer armor as well.

We design the Crash Survival Memory Unit (CSMU) with the help of CAD software that is CATIA V5.

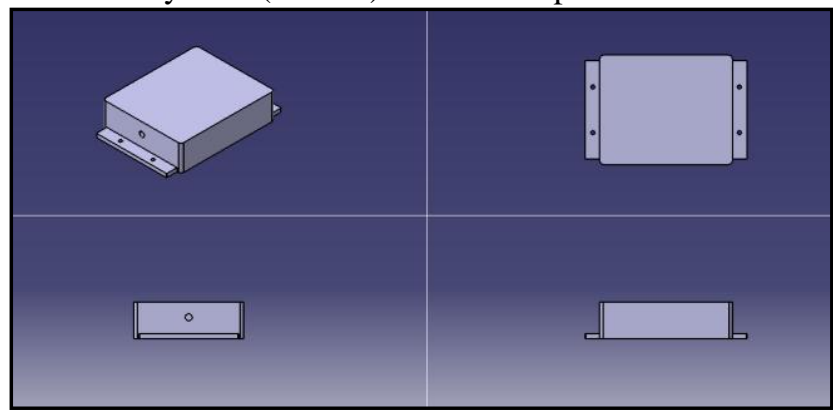

Fig.2.1 Orthographic view of CSMU

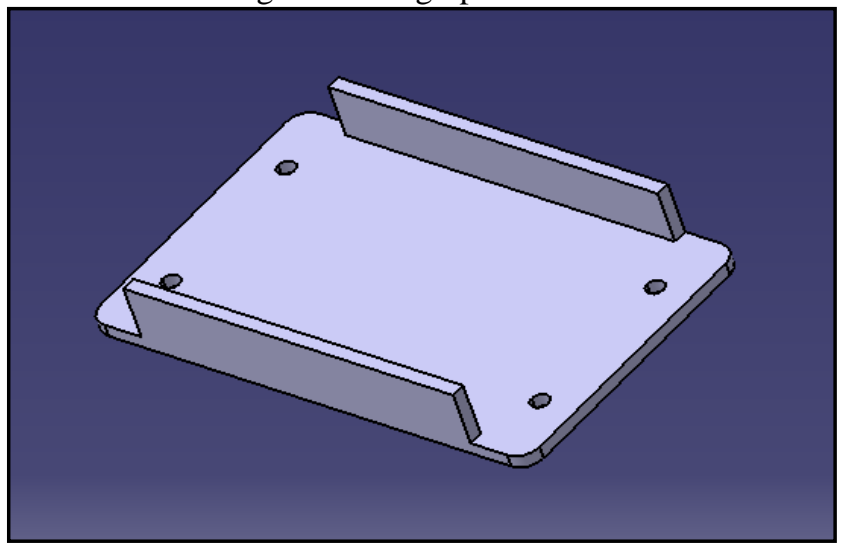

Fig.2.2 Isometric view of base plate of CSMU

\subsection{Manufacturing Process of CSMU}

The key to manufacturing a successful black box is to make it as indestructible as possible. This is done by sheathing the components inside a multi-layer protective shell. The different makers of recorders each have their own proprietary design, but in general the manufacturing process can be described as follows:

1. The key components (the power supply, the interface/controller board, and the memory circuits) are built as separate units and then assembled to form the completed black box. This modular approach allows the components to be easily replaced without disassembly of the entire device. Each of these components has its own special assembly requirements, but primary attention is given to the protecting the memory unit.

2. A multi-layered configuration is used to ensure the memory unit's integrated circuits are adequately protected. The outermost layer is the housing, which consists of steel armor plate.

3. Below that is a layer of insulation, followed by a thick slab of paraffin, which forms a thermal block. As the paraffin melts, it absorbs heat and therefore keeps the temperature of the memory core lower.

4. Beneath the paraffin lies the board containing the memory chips.

5. The assembled Crash Survivable Memory Unit is then bolted onto the front of a heavy metal plate mounting shelf with four large retaining bolts. The power supply is attached just behind the CSMU.

6. The above CSMU box shown in design is manufactured in sheet metal workshop, with forging and welding operation. To increase the strength of the CSMU, we need to cast the box.

7. For bolt fitting we used drilling operation in base plate and box.

\subsection{TESTING OF CSMU}

To ensure the quality and survivability of black boxes, we thoroughly test the CSMU. Remember, only the CSMU has to survive a crash -- if accident investigators have that, they can retrieve the information they 
need. In order to test the unit, engineers load data onto the memory boards inside the CSMU. There are several tests that make up the crash-survival sequence:

Crash impact - Researchers shoot the CSMU down air cannon to create an impact of $100 \mathrm{Gs}$ ( $1 \mathrm{G}$ is the force of Earth's gravity, which determines how much something weighs). At 100Gs, the CSMU hits aluminum, honeycomb target at a force equal to 100 times its weight. This impact force is equal to or in excess of what a recorder might experience in an actual crash.

Static crush - For five minutes, researchers apply 800 pounds per square-inch (psi) of crush force to each of the unit's six major axis points.

Fire test - Researchers place the unit into a propane-source fireball, cooking it using three burners. The unit sits inside the fire at 2,000 degrees Fahrenheit $(1,100 \mathrm{C})$ for one hour. The FAA requires that all solid-state recorders be able to survive at least one hour at this temperature.

\section{Performance Analysis}

When accident will occur vibration will generate in the body of automobile which will trigger the vibration sensor. When vibration sensor gets triggered it will automatically cut off the supply of power from the battery and also stop the recording from all the sensors. Then it will store all the numeric data inside the memory slot. When the investigation team wants to retrieve the data from memory unit they have to connect complete system with the PC. After that they have to press the retrieve button placed on the board, which will retrieve all the stored data and video recording and display on the screen of the PC in the numeric as well as graphical format as shown in figure below.

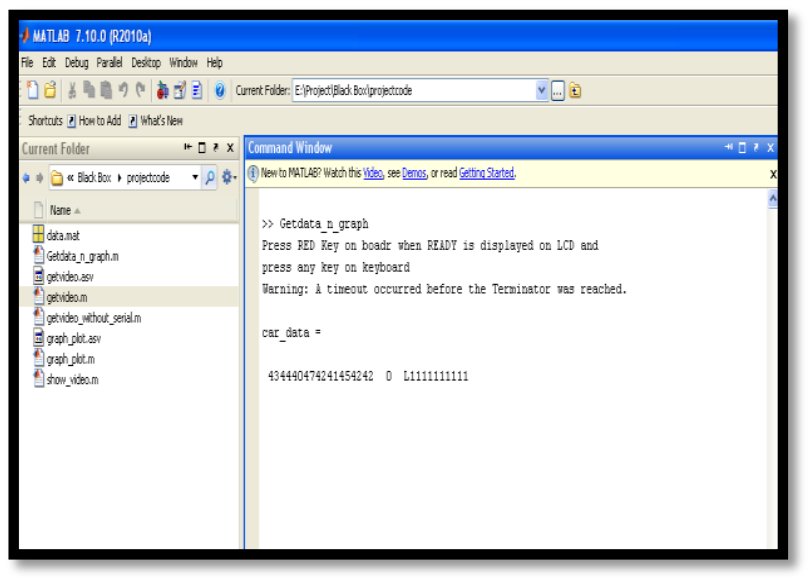

Fig 3.1.Retrieved data on MATLAB

In the above figure we can see the numeric data which is retrieved from the black box, which can be plotted in the form of graphs for the easy understanding of the situation. Depending upon the conditions there will be the changes in the numeric as well as the graphical plotting.

If we have to plot the graph on the above numeric data it will come as shown in below figure.

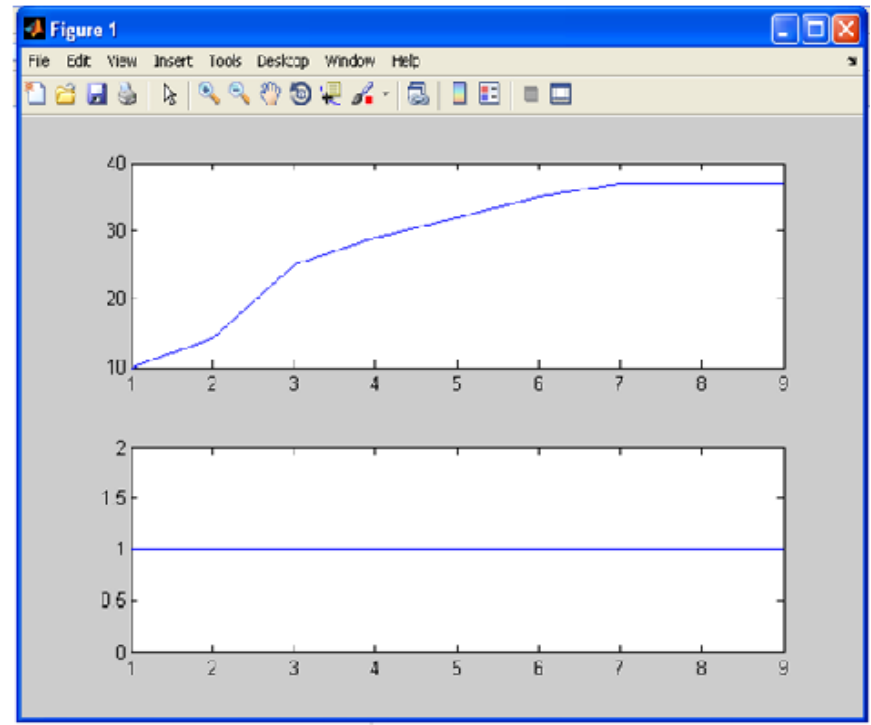

Fig 3.2.Graphical plotting (Speed and Break sensors) 
From this graph we came to know that at the time of accident driver applied brake on the level 1 which is not sufficient to break the automobile hence the speed of the automobile goes on increasing. From this we come to know that driver did not put enough effort to brake the vehicle or he was not aware of the accidental situation. More information about the road and traffic condition at the time of accident can retrieve from the video camera

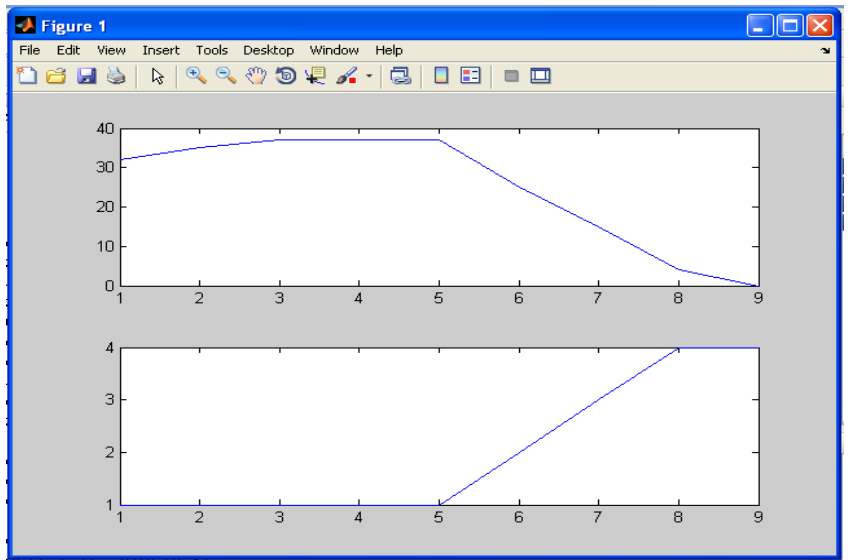

Fig3.3. Graphical plotting (Speed And Break)

From this graph we came to know that at the time of accident driver applied brake up to the level 4 which is sufficient to stop the automobile hence the speed of the automobile goes on decreasing. From this we come to know that driver put enough effort to break the vehicle or he was aware of the accidental situation. More information about the road and traffic condition at the time of accident can retrieve from the video camera.

From video recording one can easily understand what actually happened before and during the accident. In accident whether driver responsible for the accident or ahead situation? The question can get clear due to video recording.

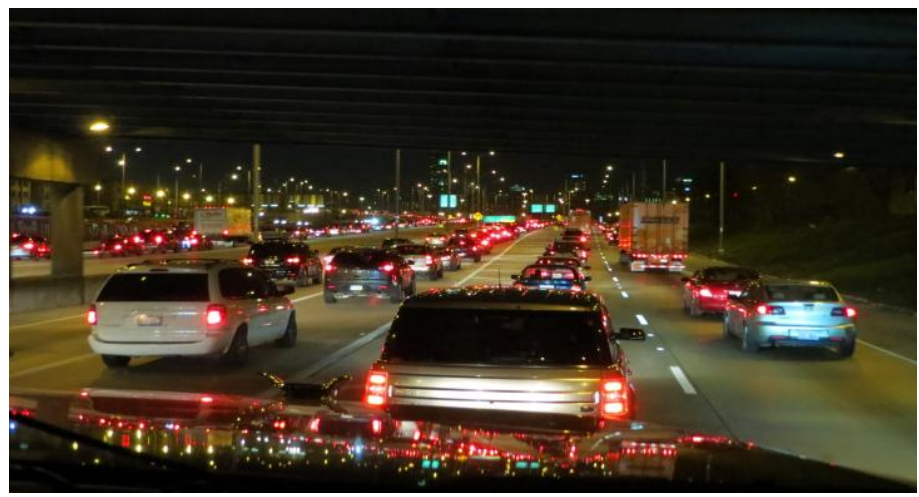

Fig 3.4.Recording from the video camera[15]

\section{Advantages \& Disadvantages:}

\subsection{Advantages}

1. Reliable device.

2.Easy to extract the data .

3.Easy to analyze the recorded data.

4.It will not affect the privacy of driver.

5.Helpful to car manufacturer to improve the car design.

6.Help to investigation team

7.Help to Insurance Company

8.If it is connected to GPS system

9.Module alerts the accident to present mobile numbers stored in it.

10.The alert message includes the location in latitude and longitude.

\subsection{Disadvantages}

1.As the black box mainly consists of electronic circuit, there are chances of

2.damage results in providing wrong data.

3.Initial cost is high.

4.Even a small damage in black box will cause to replace it, which will increase in cost. 


\section{Conclusion:}

As there are many advantages of installing black box in cars. Automotive black boxes are different than the kind that is in airplanes, which continuously record audio, mechanical functions and location. In cars, the box only transmits information to the car's computer in the event of a crash or air bag deployment. Recorders capture vehicle speed, brake activity, crash forces and even seatbelt use at the time of the event. In future the Automobile Black Box will be the integrated part of an automobile. Due to its advantages and demand of time. It helps not only investigation team but also it will be a helping hand to the insurance companies. Also it will help to those innocent drivers who are falsely charged with accident cases.

\section{Reference}

[1]“ Don Gilman”Automotive Black Box Data Recovery Systems

[2]“ Paul Black And Dylan Wiliam”Inside The Black Box

[3]“ KwangIkSeoEun Man Choi”Comparison Of Five Black-Box Testing Methods For Object-Oriented Software.

[4]" Tony Bailey"Flight Data Recorders Built To Survive. [5]"Paul Black And Dylan William”Inside The Black Box: Raising Standards

[6]" Stephen H. Edwards"A Framework For Practical, Automated Black-Box

[7] http://www.howstuffworks.com/gadgets/other-gadgets/black-box.htm

[8 ]http://spectrum.ieee.org/aerospace/aviation/beyond-the-black-box/0

[9] http://news.discovery.com/tech/beyond-the-black-box.html

[10] http://en.wikipedia.org/wiki/Flight_data_recorder

[11] http://en.wikipedia.org/wiki/Black box

[12] http://en.wikipedia.org/wiki/List of unrecovered flight recorders

[13] http://en.wikipedia.org/wiki/Emergency Position Indicating Radio Beacon

[14] http://en.wikipedia.org/wiki/Data logger.

[15] www.google.com 